\title{
Li-Fi (LIGHT FIDELITY) - THE CHANGING SCENARIO OF WIRELESS COMMUNICATION
}

\author{
Nitin Vijaykumar Swami ${ }^{1}$ \\ ${ }^{1}$ Computer Engineering Department, Shri Yogeshwari Polytechnic College, Ambajogai, Beed
}

\begin{abstract}
As per the growing demands of wireless communication there is enormous new technologies participating to make sophisticated environment for an end user. In a recent survey of International Telecommunication Union (ITU) it was found that the number of mobile phone subscribers has exceeds seven billion subscriptions at the end of 2014 using more than 10 to 15 internet utility apps which is more than the entire population of planet. To serve this increasing number and size of wireless communication system the German physicist - Harald Haas proposed a "Li-Fi technology" which he calls "Data through illumination" or "D-Light". Li$F i$ is a new approach of VLC which has much more similar working of a OFC communication system providing data rates of ten's of Gbps. In this technology data is interpreted by the LED's ON/OFF concepts.
\end{abstract}

This paper gives a brief focus on Li-Fi technology, it's working principal, some misconceptions about Li-Fi and related researches like Nobel Prize winning Blue Light emitting diode, Optoelectronic integrated circuit (OEIC) receiver, etc.

Keywords: Li- Fi, VLC, Visible light communication, photo detector, optoelectronic integrated circuit, OEIC receiver, $A P$.

\section{INTRODUCTION}

The statistics of ITU said that the use of mobile and internet is increased so that there is around 11 Exabyte $\left(10^{18}\right)$ of data transferred through mobile networks ${ }^{[1]}$. When we consider the other electronics for communication it will surely exceed some more tens of Exabytes. And also due to increase in various communication systems there is no doubt of electromagnetic spectrum band gets occupied within a few next tens of decades.

Over the past decade, significant research efforts have been directed towards exploring alternative parts of the electromagnetic spectrum that could potentially offload a large portion of the network traffic from the overcrowded RF domain ${ }^{[2]}$. The Li-Fi brings the best solution over these all problem which uses VLC. This fidelity concept is much more similar or you may call it as combination of OFC and Wi-Fi by adding and removing few elements.

It works on LED's ON/OFF concept encoded binary 'one' for light ON and binary 'zero' for light OFF condition. As we know the speed of light is about 1080 million $\mathrm{km} / \mathrm{hr}$ and we are using the light source for data transmission, we can achieve the data rates in the range of Gbps. From the current experiments, the Li-Fi consortium believes it is possible to achieve more than $10 \mathrm{Gbps}$ speed, theoretically allowing a high definition film to be downloaded in 30 seconds ${ }^{[3]}$.

\section{WORKING PRINCIPLE OF LI-FI:}

The working of Li-Fi is based on VLC, which uses visible light for data transmission. The visible light spectrum has wider range of hundreds of $\mathrm{THz}$ of free bandwidth, which is
10,000 times more than RF spectrum up to $30 \mathrm{GHz}$. It uses LED to generate data stream which is connected to the internet or cellular system. As per the data stream the LED flickers at high rate which is not recognized by human eye. 
The fig. 1 shows the working principle of a Li-Fi.

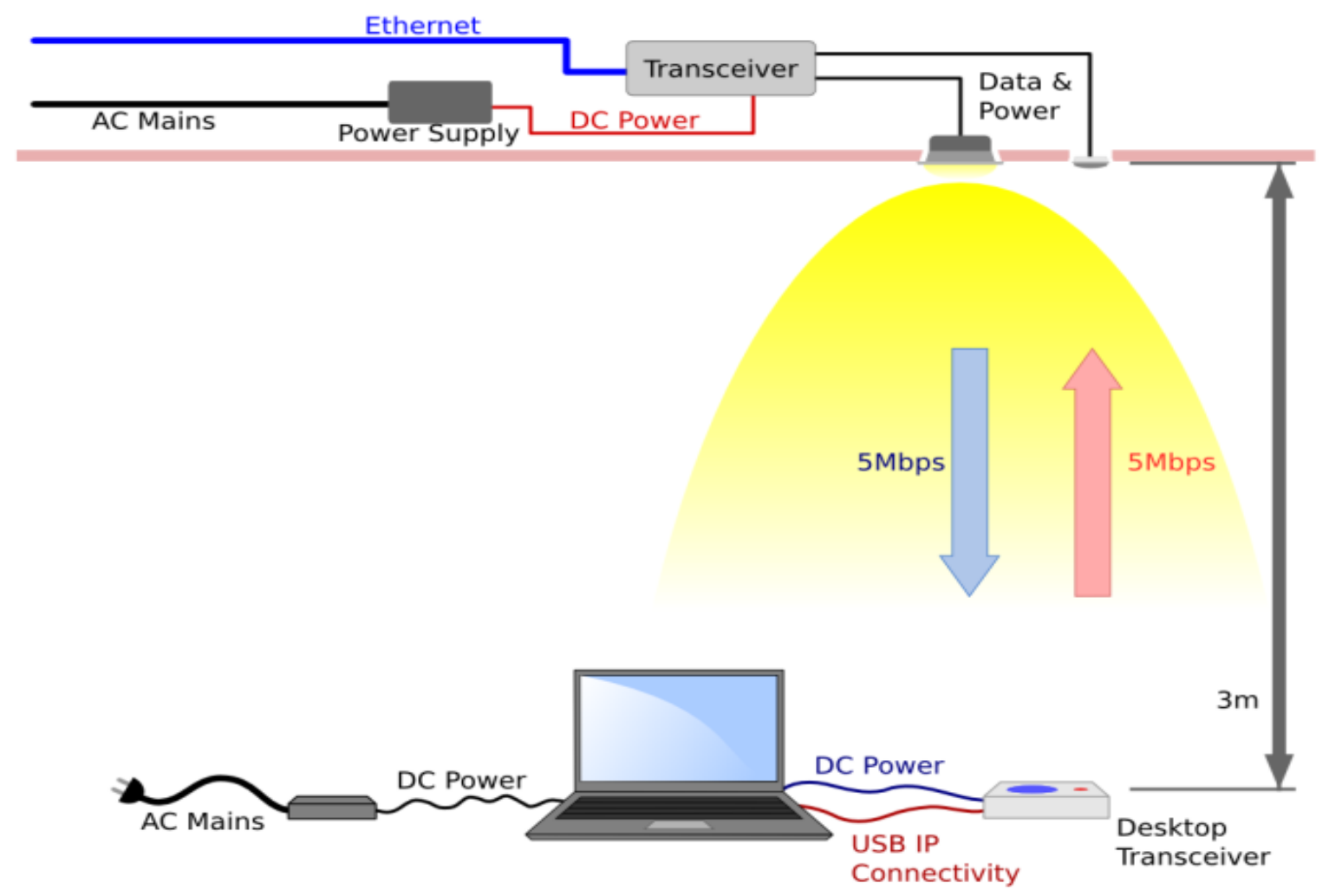

Fig. 1: Working Principle of LI-FI Technology

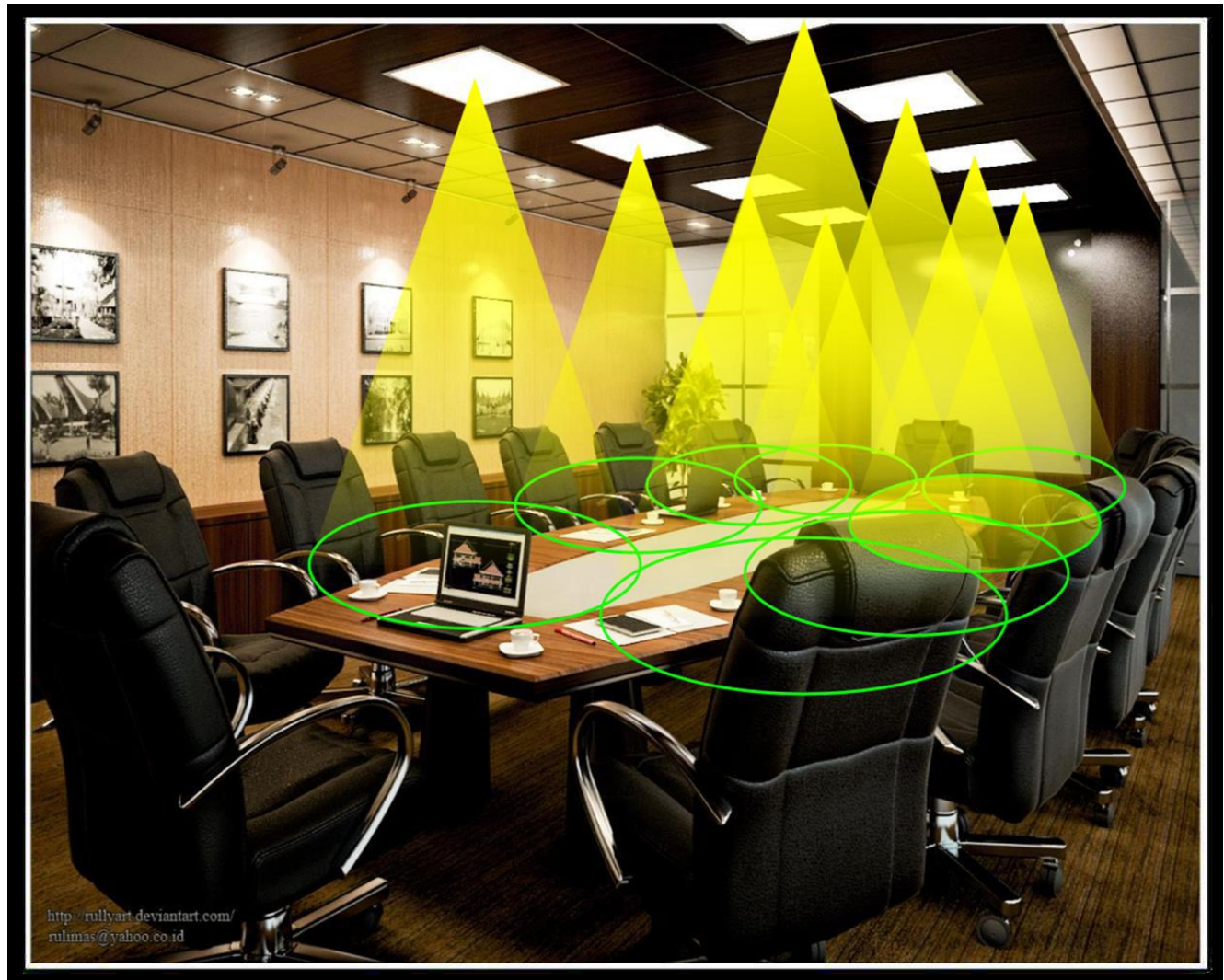

Fig. 2: Multiple Li-Fi APs in a room 
The flickering of LED is regulated by voltage regulator and level shifter circuit. At the receiver side of the system photo detector is used ${ }^{[4]}$. This photo detector senses light and converts into the respected pulses. These pulses are then amplified and processed to achieve the original data stream.

The distance achieved by system depends only on the potential of the light source, that is LED lamp. The one among the major advantage of $\mathrm{Li}-\mathrm{Fi}$ is the merging of illumination with wireless communication provides a measurable reduction in both infrastructure complexity and energy consumption ${ }^{[8]}$.

\section{LI-FI SOURCE - LED}

As LED is more commonly used source for room lighting, it is also used in $\mathrm{Li}-\mathrm{Fi}$ as a data source more sophisticatedly and efficiently to generate data streams. As compare to the IR LED which generate a single data stream with 10-20 kbps speed, these LED's generates a thousands of data streams spreading all over the room where the light can reach with a very fast rate ${ }^{[5]}$. The potential of these LED's can be increased by using some Luminaire Design Optimization techniques.

Recently the R \& D centre of pure VLC has achieved $3.5 \mathrm{Gbps}$ of data rate from a single color micro LED operating at $5 \mathrm{~mW}$ with a $1 \mathrm{~m}$ distance and $1.1 \mathrm{Gbps}$ of data rate at $10 \mathrm{~m}$ at $5 \mathrm{~mW}^{[5]}$. So it can be concluded that by using three colors RGB LED's the data rates of more than 10Gbps can be achieved.

\section{UPLINK}

For a complete Li-Fi communication system, full duplex communication is required, that is an uplink connection from mobile terminals to the optical AP has to be provided [1]. This can be achieved by using the infrared (IR) transmission, where the mobile terminals can send data using infrared frequency and optical AP receives it to respond. The first commercially available full duplex Li-Fi modem using IR light has been announced by pure $\mathrm{Li}^{-\mathrm{Fi}^{[6]}}$.

\section{LI-FI RECEIVER - PHOTODIODE}

The photodiode is used as a $\mathrm{Li}-\mathrm{Fi}$ receiver to sense the data stream. The Avalanche photodiodes are used to make better receivers. At Haas's recent talk on "My Li-Fi Revolution" at Tam Dalyell prize lecture he shown the first receiver chip for Li-Fi with integrated Avalanche photodiodes on CMOS created by his team at the Li-Fi R\&D centre ${ }^{[5]}$. The $7.8-$ square-mm IC houses 49 photodiodes ${ }^{[7]}$ which is around $13 \%$ of length of single Wi-Fi antenna.

At the IEEE Photonics Conference in October, Li-Fi consortium showed off the progress of combining both emitters and photodiodes to detect light using available red, green and blue LED's. By doing that the system could both send and receive data at aggregate rates of $110 \mathrm{Mbps}^{[7]}$.

\section{RELATED RESEARCHES FOR IMPROVING}

\section{LI-FI SYSTEM}

\subsection{Nobel Prize in Physics 2014 - Efficient Blue}

\section{Light - Emitting Diodes}

Presently, there are two approaches to creating white light. Mixed-color white light which mixes the multicolor LED's and Phosphor-converted white light which uses phosphors together with a short-wavelength $\mathrm{LED}^{[9]}$. The three scientists Isamu Akasaki, Hiroshi Amano and Shuji Nakamura produced bright blue light beams from their semi-conductors in the early 1990s, they triggered a fundamental transformation of lighting technology. Red and green diodes had been around for a long time but without blue light, white lamps could not be created. Despite considerable efforts, both in the scientific community and in industry, the blue LED had remained a challenge for three decades ${ }^{[10]}$.

They succeeded in creating a bright source LED using excitation of a phosphor so that the blue light is converted to white light. For their achievement they are rewarded by Nobel Prize in Physics in 2014. This LED can be efficiently used as a Li-Fi source and reduces the cost of emerging LiFi market by having very long lifetime (100 000 hours).

\subsection{Opto Electronic Integrated Circuit (OEIC)}

\section{Receiver}

For improvisation of optical receiver scientist proposed optoelectronic integrated circuit (OEIC) receiver that has been analyzed theoretically. A simplified noise model of the receiver has also been developed. Results have been presented for an OEIC receiver based on InGaAs MESFET supposed to be fabricated with matured InGaAs-InP MMIC technology. Theoretical results based on a simplistic noise model reveal that the proposed OEIC receiver has superior performance characteristics over the existing optical receivers $^{[11]}$.

\section{APPLICATIONS \& ADVANTAGEOUS OF LI-}

FI:

The Various applications of $\mathrm{Li}-\mathrm{Fi}$ system providing a reliable, secure, cheaper and ultra-high-speed communication infrastructure are as follows:

a) Smart Lighting: The Li-Fi transmitter is a great combination of data source producing thousands of data streams as well as light source producing a much brighter light. So it can be effectively equipped with street lamps serving multiple users and multiple users can talk to street lamps.

b) In Vehicles Light: We can use the Li-Fi in vehicles head lights and back lights developing an intelligent transport system where cars can talk to each other and also to the traffic lights providing statistics.

c) Undersea Water: Radio Frequencies can't propagate undersea water as its salty, high conductivity and due to a high attenuation but light does. Cables are 
creating threads in communication undersea water so they can be replaced with Li-Fi transmitters by which communication is freer to explore. Also they can send data to submarines, to surface as well as to divers with their head lights.

d) In Home Appliances: Li-Fi system can be integrated in home appliances like thermos, freeze, clocks, TV's etc. connecting to an Internet to reduce energy consumptions for intelligent energy management.

e) In Health: The wearable Li-Fi transmitter like LED ear rings, wrist watches etc. can allow to monitor your health issues more sophisticatedly and providing any health concern changes to your family doctors by connecting to the internet and updating your information online.

f) Indoor Navigation: The Li-Fi system allows you for an indoor navigation where the LED lights sources are used like shopping malls, cinema theatres, government offices, work offices or any indoor locations.

g) In Hospitals: The Li-Fi transmitters can be enhanced in hospitals also where the RF wave does not. They can be used in different machines to communicate with each other for fast data interpretation.

h) In Petrochemical Industries: These industries do not allow RF because various radioactive chemicals are used for processing. The Li-Fi system can be used here as it uses a VLC.

i) Security: As the visible light cannot penetrates the walls the security can be achieved at higher levels.

\section{COMMON MISCONCEPTIONS}

The common misconceptions raised about $\mathrm{Li}-\mathrm{Fi}$ are as follows:

a) Lights cannot be dimmed: The experiments with a various dimmed intensities are observed at $\mathrm{Li}-\mathrm{Fi}$ R\&D centre and they have found that there no effect of deemed light.

b) The lights flickers: The Li-Fi light flickers at higher rates which is not recognizable and hazardous to the human eye. The human eye can sense flickering of lower rates that is below $120-150 \mathrm{~Hz}$.

c) This is for downlink only: As discussed earlier the Li-Fi system uses IR light for uplink completing the full duplex communication.

d) There will be interference from sunlight: The Li-Fi light sources uses visible light having a different intensity level and there are various energy harvesting techniques are present that can be effectively used to improve the Li-Fi system.

e) Lights need to be on so this is insufficient: The LiFi system will be using the existing infrastructure of light distribution; we need to just update this with LiFi equipments. So, it reduces the existing cellular system, the maintenance cost of it, the space required and the power requirements of it.

f) This is a line-of-sight technology: As the demonstration of $\mathrm{Li}-\mathrm{Fi}$ at Edinburgh University lecture Haas had shown that this system can work beyond the line of sight as photo detectors are that much of smart to sense the light.

\section{CONCLUSION}

As the electromagnetic spectrum shrinking continuously the Li-Fi system will going to provide a greener, safer, better and healthier future for communication system. When this system will be developed each light source can be used as a Li-Fi AP means where is a light there is a Internet. Also it will shapes the better future for human kind by reducing the energy consumption, data as well as light at low cost, minimal cellular infrastructure and creating the employments opportunities at large scale. In short the Li-Fi system will be going to change the scenario of wireless communications in many greener ways.

\section{REFERENCES}

[1] Cisco Visual Networking Index, "Global Mobile Data traffic Forecast Update 2012-2017," White paper, CISCO.

[2] Dobroslav Tsonev, Stefan Videv and Harald Haas, "Light Fidelity (Li-Fi): Towards All-Optical Networking", Institute for Digital Communications, Li-Fi R\&D Centre, Edinburgh, UK.

[3] Jyoti Rani, Prerna Chauhan, Ritika Tripathi, "Li-Fi (Light Fidelity)-The future technology In Wireless communication", in International Journal of Applied Engineering Research, ISSN 0973-4562 Vol.7 No.11 (2012)

[4] Jay H. Bhut, Dharmrajsinh N. Parmar, Khushbu V. Mehta, "LI-FI Technology - A Visible Light Communication"in INTERNATIONAL JOURNAL OF ENGINEERING DEVELOPMENT AND RESEARCH (RTEECE-2014 -17th ,18th January 2014)

[5] Video.fromhttps://www.youtube.com/watch?v=WR G9iXZbuAc by Harald Haas on 'My Li-Fi Revolution'

[6] pureVLC, "pureVLC Li-1st." video. http://purevlc.co.uk/li-fire/purevlc-li-1st/.

[7] http://spectrum.iee.org/tlecom/internet/lifi-getsready-to-compete-with-wifi

[8] http://purelifi.com/li-fire/li-flame/

[9] http://www.lrc.rpi.edu/programs/nlpip/lightinganswer s/led/whiteLight.asp

[10] http://www.nobelprize.org/nobel_prizes/physics/laur eates/2014/press.html

[11] http://ieeexplore.ieee.org/xpl/articleDetails.jsp 